\title{
Experimental Organism Intramural Schwannoma
}

National Cancer Institute

\section{Source}

National Cancer Institute. Experimental Organism Intramural Schwannoma. NCI

Thesaurus. Code C156608.

A malignant schwannoma of the heart of the heart arising from intramural swan cells that appears as a poorly circumscribed spindle cell mass within the ventricular myocardium with a more discrete boundary, that is generally more infiltrative than expansile. (INHAND) 